\title{
Outcomes of stereotactic radiosurgery for hemorrhagic arteriovenous malformations with or without prior resection or embolization
}

\author{
Mariko Kawashima, MD, ${ }^{1}$ Hirotaka Hasegawa, MD, PhD, ${ }^{1,2}$ Masahiro Shin, MD, PhD, ${ }^{1}$ \\ Yuki Shinya, MD, ${ }^{1}$ Osamu Ishikawa, MD, PhD, ${ }^{1}$ Satoshi Koizumi, MD, ${ }^{1}$ Atsuto Katano, MD, PhD, ${ }^{3}$ \\ Hirofumi Nakatomi, MD, PhD, ${ }^{1}$ and Nobuhito Saito, MD, PhD1 \\ Departments of ${ }^{1}$ Neurosurgery and ${ }^{3}$ Radiology, University of Tokyo Hospital, Tokyo, Japan; and ${ }^{2}$ Department of Neurologic \\ Surgery, Mayo Clinic, Rochester, Minnesota
}

\begin{abstract}
OBJECTIVE The major concern about ruptured arteriovenous malformations (rAVMs) is recurrent hemorrhage, which tends to preclude stereotactic radiosurgery (SRS) as a therapeutic modality for these brain malformations. In this study, the authors aimed to clarify the role of SRS for rAVM as a stand-alone modality and an adjunct for a remnant nidus after surgery or embolization.
\end{abstract}

METHODS Data on 410 consecutive patients with rAVMs treated with SRS were analyzed. The patients were classified into groups, according to prior interventions: SRS-alone, surgery and SRS (Surg-SRS), and embolization and SRS (Embol-SRS) groups. The outcomes of the SRS-alone group were analyzed in comparison with those of the other two groups.

RESULTS The obliteration rate was higher in the Surg-SRS group than in the SRS-alone group (5-year cumulative rate $97 \%$ vs $79 \%, p<0.001$ ), whereas no significant difference was observed between the Embol-SRS and SRS-alone groups. Prior resection (HR 1.78, 95\% Cl 1.30-2.43, p < 0.001), a maximum AVM diameter $\leq 20 \mathrm{~mm}(\mathrm{HR} 1.81,95 \% \mathrm{Cl}$ $1.43-2.30, p<0.001)$, and a prescription dose $\geq 20 \mathrm{~Gy}(\mathrm{HR} 2.04,95 \% \mathrm{Cl} 1.28-3.27, \mathrm{p}=0.003)$ were associated with a better obliteration rate, as demonstrated by multivariate Cox proportional hazards analyses. In the SRS-alone group, the annual post-SRS hemorrhage rates were $1.5 \%$ within 5 years and $0.2 \%$ thereafter and the 10 -year significant neurological event-free rate was 95\%; no intergroup difference was observed in either outcome. The exclusive performance of SRS (SRS alone) was not a risk for post-SRS hemorrhage or for significant neurological events based on multivariate analyses. These results were also confirmed with propensity score-matched analyses.

CONCLUSIONS The treatment strategy for rAVMs should be tailored with due consideration of multiple factors associated with the patients. Stand-alone SRS is effective for hemorrhagic AVMs, and the risk of post-SRS hemorrhage was low. SRS can also be favorably used for residual AVMs after initial interventions, especially after failed resection. https://thejns.org/doi/abs/10.3171/2020.7.JNS201502

KEYWORDS Gamma Knife; long-term outcomes; ruptured arteriovenous malformation; stereotactic radiosurgery; vascular disorders

A DVANCES in imaging studies and improved access to these developments have led to an increase in the incidental diagnosis of brain arteriovenous malformation (AVM); however, hemorrhagic stroke remains the prominent reason for its diagnosis..$^{1,2}$ In addition, hemorrhage due to rupture is associated with mortality and morbidity rates of up to $29 \%$ and $34 \%$, respectively. ${ }^{3-6}$ It is well known that the risk of future bleeding increases in AVMs with a history of bleeding. ${ }^{7-9}$ Because of this, therapeutic interventions for ruptured AVMs (rAVMs) are widely considered reasonable, although recent prospective studies have demonstrated skepticism regarding the safety of prophylaxis interventions for unruptured AVMs..$^{10,11}$

Stereotactic radiosurgery (SRS) effectively obliterates the nidus by inducing endothelial damage followed by the progressive thickening of intimal layers and the subsequent thrombosis of nidal vessels over a couple of years. ${ }^{12} \mathrm{Al}-$ though SRS is a reasonable minimally invasive therapeutic option for small to medium AVMs, some argue that a period of latency before obliteration of the nidus would pose

ABBREVIATIONS AVM = arteriovenous malformation; DSA = digital subtraction angiography; mRBAS = modified radiosurgery-based AVM score; $m$ RS = modified Rankin Scale; $r A V M=$ ruptured AVM; RIC = radiation-induced change; SMG = Spetzler-Martin grade; SNE = significant neurological event; SRS = stereotactic radiosurgery; VRAS = Virginia Radiosurgery AVM Scale.

SUBMITTED April 28, 2020. ACCEPTED July 13, 2020.

INCLUDE WHEN CITING Published online December 4, 2020; DOI: 10.3171/2020.7.JNS201502. 
a certain risk of stroke, especially in rAVM cases, ${ }^{13,14}$ and thus resection or endovascular treatment is preferred. Accordingly, in cases of rAVMs, SRS has been mainly used for posttreatment remnants or rAVMs in deep brain locations for which surgical intervention is challenging. In other words, SRS alone for rAVM has been scarcely highlighted, and actual outcomes have yet to be elucidated. Therefore, in this study, we aimed to assess the efficacy and safety of SRS for rAVM as a stand-alone modality and as an adjunctive treatment following resection and embolization.

\section{Methods}

\section{Patient Selection}

Of 3064 procedures (in the SRS database) performed between 1990 and 2016 at our institution, 512 procedures in 475 patients with rAVMs were identified. AVMs with radiographic findings suggesting previous hemorrhage (hemosiderin deposition or cavity formation) without clinical signs or symptoms were not considered true rAVMs. After exclusions for planned volume-staged SRS $(n=28)$, a history of previous radiation $(n=7)$, and no radiological follow-up ( $\mathrm{n}=30)$, a total of 410 patients who had undergone 411 initial radiosurgeries for rAVMs were included in our study. Notably, 1 patient had 2 separate rAVMs treated with SRS alone.

The patients were classified into three groups, depending on their interventions before SRS: 1) SRS alone, 2) surgery and SRS (Surg-SRS), and 3) embolization and SRS (Embol-SRS). Surgery was defined as any direct surgical intervention to the nidus; hematoma evacuation without intervention to the nidus, ventricular drainage, and clipping for a feeder aneurysm were excluded. Patients who had undergone resection after embolization were allocated to the Surg-SRS group. Embolization was defined as any endovascular embolization to the nidus, feeders, or intranidal aneurysm; however, embolization to a flow-related aneurysm was excluded. This study was approved by the clinical research review board at the University of Tokyo, and all participants provided written informed consent at the time of treatment.

\section{Radiosurgical Techniques and Posttreatment Follow-Up}

The Leksell Gamma Knife (Elekta AB) was used in all cases. Detailed procedures were described in previous articles. ${ }^{14-16}$ Digital subtraction angiography (DSA) was solely used as the stereotactic imaging modality before February 1991; thereafter, CT (March 1991-July 1996) and MRI (August 1996-2016) were used in combination to increase planning accuracy. Radiosurgical plans were created with commercially available planning software (KULA until 1998 and Leksell GammaPlan thereafter; Elekta AB). A prescription dose of 20 Gy was selected for most cases, although the doses were lowered in cases of large AVMs (usually $>10 \mathrm{ml}$ in volume) and eloquent AVMs based on the surgeons' discretion. The patients were clinically and radiographically followed up with MRI every 6 months before obliteration and every year thereafter. Nidus obliteration was confirmed with DSA, although MRI was alternatively used when DSA was contraindicated or rejected.

Post-SRS hemorrhage was defined as any radiographi- cally confirmed intracranial hemorrhage that had occurred within or adjacent to the nidus and caused clinical symptoms. Radiation-induced changes (RICs) were defined as peripheral edema that was shown as a hyperintensity or hypoattenuation area on T2-weighted MRI or CT, respectively. Delayed adverse events such as cyst formation, encapsulated hematoma, and tumorigenesis within an irradiated field were all identified as such. ${ }^{17}$ A significant neurological event (SNE) was defined as any event causing a > 1-point decline in the modified Rankin Scale (mRS) score and associated with SRS, further treatments for the AVM, or post-SRS hemorrhage. The radiographic results were assessed not only by the attending neurosurgeons but also by radiologists at our institution in a blinded manner, whereas the clinical assessment was performed only by the attending neurosurgeons.

\section{Statistical Analysis}

First, baseline characteristics of the patients during SRS were summarized and compared among the groups using Fisher's exact test and the Mann-Whitney U-test for categorical and continuous variables, respectively. The outcomes of prior treatments had been prospectively recorded, and changes in the mRS score were retrospectively evaluated. The modified radiosurgery-based AVM score (mRBAS), ${ }^{18}$ Spetzler-Martin grade (SMG), ${ }^{19}$ and Virginia Radiosurgery AVM Scale (VRAS) score ${ }^{20}$ were calculated based on their definitions.

Next, the nidus obliteration, post-SRS hemorrhage, and SNE-free rates were calculated with the Kaplan-Meier method and compared between groups using the logrank test. The post-SRS hemorrhage rates were similarly evaluated using the person-year method, with the period categorized in two phases-within 5 years from SRS (latency phase) and thereafter. Factors potentially affecting the rates, as mentioned earlier, were evaluated using the Cox proportional hazards model for bivariate and multivariate analyses. Continuous variables were roughly dichotomized with their median values. The factors entered into the multivariate model were selected using the forward stepwise selection method with a cutoff $\mathrm{p}$ value of 0.25 . RICs were evaluated as crude rates and compared using Fisher's exact test and logistic regression analysis for bivariate and multivariate analyses, respectively.

Potential differences between groups can lead to significant biases, and to address this issue, propensity score matching was performed as a sensitivity analysis: 1) SRSalone group versus Surg-SRS group and 2) SRS-alone group versus Embol-SRS group. Propensity scores were generated using a binary logistic regression model with the following variables: sex, patient age at SRS, maximum diameter and volume of AVM, deep drainage, eloquent location, mRBAS, SMG, and VRAS score. Thereafter, one-to-one matching without any replacement was completed using the nearest neighbor match with a caliper of 0.05. The obliteration rates, post-SRS hemorrhage rates, and SNE-free rates were evaluated with the Kaplan-Meier method and log-rank test.

A p value $<0.05$ was considered statistically significant. All analyses were performed using JMP Pro 14 software (SAS Institute Inc.). 
TABLE 1. Baseline patient and AVM characteristics

\begin{tabular}{|c|c|c|c|c|c|}
\hline Variable & Overall & SRS-Alone Group & Surg-SRS Group & Embol-SRS Group & p Value* \\
\hline No. of cases & 411 & 306 & 60 & 45 & \\
\hline Male sex, no. (\%) & $217(53)$ & $166(54)$ & $30(50)$ & $21(47)$ & $0.572 / 0.341$ \\
\hline Median age at SRS in yrs (range) & $28(4-80)$ & $30(4-80)$ & $24(8-64)$ & $28(5-76)$ & $0.113 / 0.681$ \\
\hline Significant complications prior to SRS, $†$ no. (\%) & & & $6(10)$ & $5(11)$ & \\
\hline \multicolumn{6}{|l|}{ mRS score at SRS, no. (\%) } \\
\hline $0-2$ & $353(86)$ & $270(88)$ & $47(78)$ & $36(80)$ & \multirow{2}{*}{$0.032 / 0.871$} \\
\hline $3-5$ & $58(14)$ & $36(12)$ & $13(22)$ & $9(20)$ & \\
\hline Median max diameter in mm (range) & $19(3-68)$ & $19(3-68)$ & $17(7-48) \ddagger$ & $25(9-60) \ddagger$ & $0.143 /<0.001$ \\
\hline Median nidus vol in ml (range) & $1.4(0.1-44.5)$ & $1.4(0.1-23.5)$ & $1.0(0.1-16.7) \ddagger$ & $4.1(0.2-44.5) \ddagger$ & $0.052 /<0.001$ \\
\hline Median prescription dose in Gy (range) & $20(10-28)$ & $20(10-28)$ & $20(18-25.2)$ & $20(17-25.2)$ & $0.014 / 0.399$ \\
\hline Median mRBAS (range) & $0.97(0.18-5.47)$ & $0.99(0.18-3.28)$ & $0.74(0.25-2.13)$ & $1.06(0.37-5.47)$ & $<0.001 / 0.242$ \\
\hline Eloquent location, no. (\%) & $277(67)$ & $213(70)$ & $33(55)$ & $32(71)$ & $0.036 / 0.864$ \\
\hline Deep drainage, no. (\%) & $292(71)$ & $228(75)$ & $34(57)$ & $30(67)$ & $0.007 / 0.280$ \\
\hline \multicolumn{6}{|l|}{ SMG, no. (\%) } \\
\hline$|-| \mid$ & $171(42)$ & $121(40)$ & $33(55)$ & $17(38)$ & \multirow{2}{*}{$0.032 / 0.871$} \\
\hline$\geq|I|$ & $240(58)$ & $185(60)$ & $27(45)$ & $28(62)$ & \\
\hline \multicolumn{6}{|l|}{ VRAS score, no. (\%) } \\
\hline $1-2$ & $258(63)$ & $195(64)$ & $45(75)$ & $18(40)$ & \multirow{2}{*}{$0.103 / 0.003$} \\
\hline $3-4$ & $153(37)$ & $111(36)$ & $15(25)$ & $27(60)$ & \\
\hline
\end{tabular}

Boldface type indicates statistical significance $(p<0.05)$.

* A p value for the SRS-alone versus Surg-SRS groups/SRS-alone versus Embol-SRS groups.

$\dagger$ Those causing a > 1-point decline in the mRS score were considered significant.

$\ddagger$ Data at the time of SRS.

\section{Results}

\section{Baseline Characteristics}

Baseline characteristics are summarized in Table 1. The SRS-alone, Surg-SRS, and Embol-SRS groups comprised $306(74.5 \%), 60(14.6 \%)$, and $45(10.9 \%)$ cases, respectively. The median follow-up periods were 111 months (range 1-351 months), 121 months (5-317 months), and 79 months (13-325 months) in the SRS-alone, Surg-SRS, and EmbolSRS groups, respectively. The median intervals from hemorrhage to SRS were 3.7 months, 10.6 months ( 0.4 months from hemorrhage to initial resection), and 8.7 months (2.0 months from onset to initial embolization) in the SRSalone, Surg-SRS, and Embol-SRS groups, respectively. The median intervals from the completion of prior interventions to SRS were 10.1 and 3.4 months in the Surg-SRS and Embol-SRS groups, respectively. Persistent complications of preceding treatments causing a $>1$-point decline in the mRS score were observed in $6(10 \%)$ and $5(11 \%)$ cases in the Surg-SRS and Embol-SRS groups, respectively.

Compared to cases in the Surg-SRS group, cases in the SRS-alone group were more likely to develop deep drainage $(57 \%$ vs $75 \%, p=0.007)$ and be treated with slightly lower marginal doses $(p=0.014)$. No difference in nidus volume was observed between the SRS-alone and SurgSRS groups $(p=0.052)$. Compared to cases in the EmbolSRS group, cases in the SRS-alone group were more likely to have a smaller nidus and a smaller maximum diameter (median nidus volume $4.1 \mathrm{vs} 1.4 \mathrm{ml}, \mathrm{p}<0.001$; median maximum diameter 25 vs $19 \mathrm{~mm}, \mathrm{p}<0.001$ ).

\section{Nidus Obliteration}

Among the entire cohort, nidus obliteration was confirmed in 296 (72\%) cases at a median of 25 months after SRS, $260(88 \%)$ of whom underwent DSA. The cumulative obliteration rates were $61 \%$ and $81 \%$ at 3 and 5 years, respectively (Fig. 1A). Thirty-two patients underwent secondary SRS at a median of 53 months after the initial treatment; of these patients, nidus obliteration was confirmed in 22 , yielding a final crude obliteration rate of $77 \%$.

The cumulative 5-year obliteration rates were 79\%, 97\%, and 75\% in the SRS-alone, Surg-SRS, and EmbolSRS groups, respectively (Fig. 1B). The rate was significantly higher in the Surg-SRS group than in the SRS-alone $(\mathrm{p}<0.001)$ and Embol-SRS $(\mathrm{p}=0.003)$ groups; however, no difference was observed between the SRS-alone and Embol-SRS groups $(p=0.544)$. The multivariate Cox proportional hazards analyses demonstrated that prior resection (HR 1.78, 95\% CI 1.30-2.43, p <0.001), a maximum AVM diameter $\leq 20 \mathrm{~mm}$ (HR 1.81, 95\% CI 1.43-2.30, $\mathrm{p}$ $<0.001$ ), and a prescription dose $\geq 20$ Gy (HR 2.04, 95\% CI $1.28-3.27, \mathrm{p}=0.003$ ) were associated with a higher obliteration rate (Table 2).

\section{Post-SRS Hemorrhage}

Post-SRS hemorrhage was observed in 26 cases $(6.3 \%)$ either within 5 years after SRS (20 patients) or thereafter (6 patients), yielding an annual post-SRS hemorrhage rate of $1.2 \%$ within 5 years after SRS and $0.2 \%$ thereafter. The annual post-SRS hemorrhage rates were $1.5 \%$ in 


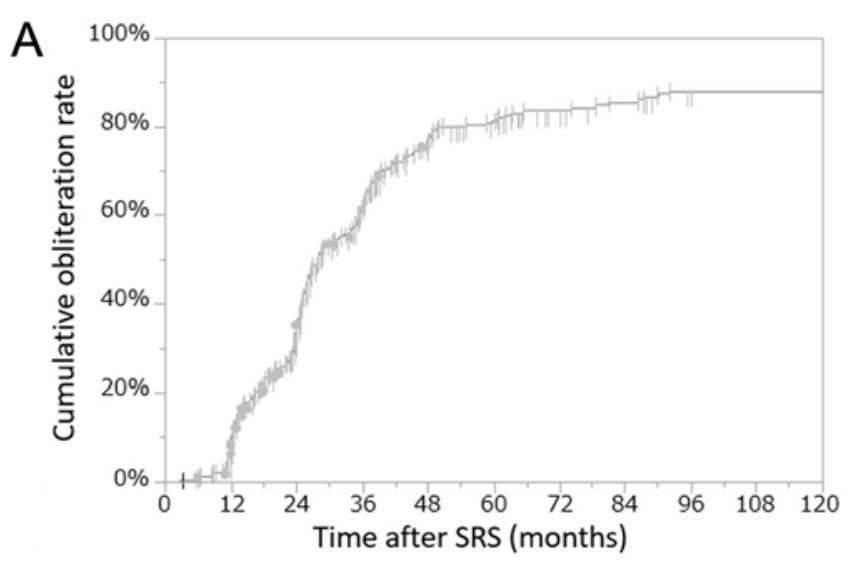

\begin{tabular}{llllllllllll}
\hline $\begin{array}{l}\text { Number at } \\
\text { risk }\end{array}$ & 411 & 361 & 235 & 128 & 66 & 46 & 30 & 24 & 16 & 15 & 15
\end{tabular}

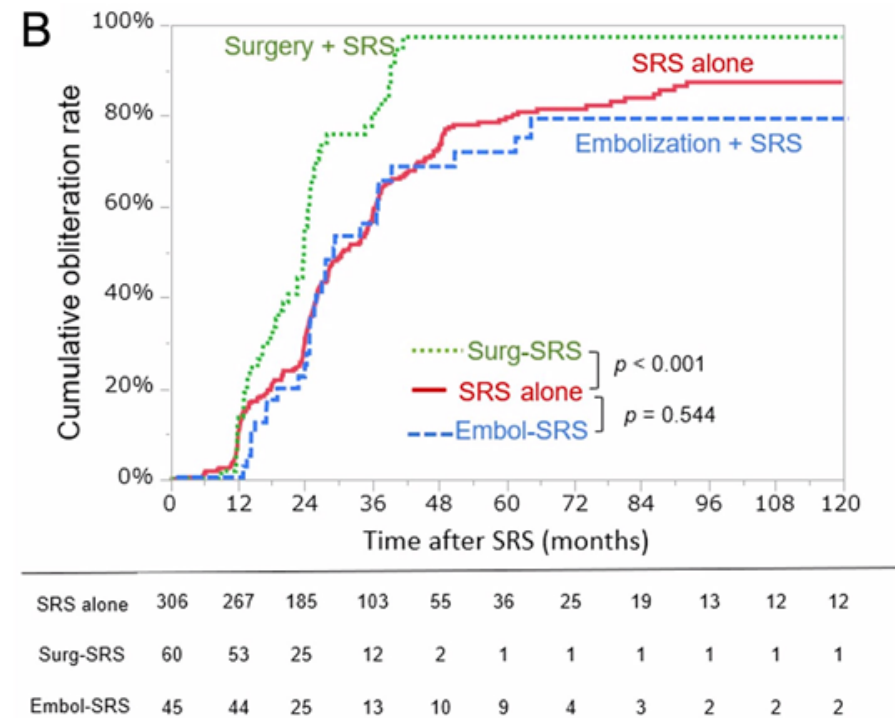

FIG. 1. Kaplan-Meier curves showing cumulative obliteration rates after initial SRS for the entire cohort (A) and between groups before propensity score matching (B). Figure is available in color online only. the SRS-alone group (19 patients over 1270 patient-years), $0 \%$ in the Surg-SRS group (0 patients over 278 patientyears), and $0.6 \%$ in the Embol-SRS group (1 patient over 165 patient-years) within 5 years after SRS; however, the rates were $0.2 \%$ in the SRS-alone group (4 patients over 2261 patient-years), $0.2 \%$ in the Surg-SRS group (1 patient over 523 patient-years), and $0.3 \%$ in the Embol-SRS group (1 patient over 349 patient-years) thereafter.

The log-rank tests showed no significant difference in the cumulative hemorrhage rates between groups (Fig. 2). The multivariate Cox proportional hazards analysis demonstrated that a prescription dose $\geq 20$ Gy (HR $0.31,95 \%$ CI $0.13-0.73, \mathrm{p}=0.007$ ) was associated with a lower hemorrhage rate (Table 3 ).

Among the patients with post-SRS hemorrhage, 7 died, 2 were severely disabled, 6 were moderately disabled (2-point decline in the mRS score), 5 developed mild functional disturbance (1-point decline in the mRS score), and 6 developed transient or no functional disturbance. PostSRS hemorrhage occurred in 2 patients following nidus obliteration; one case was caused by a de novo nidus adjacent to the original one, which triggered a mild neurological decline. ${ }^{21}$ The other case occurred from an unknown etiology but at the same location as the original nidus, resulting in no functional decline.

\section{Radiation-Induced Adverse Events and Neurological Outcomes After SRS}

In the entire cohort, symptomatic RICs were observed in 28 cases $(6.8 \%)$, consisting of 22 patients $(7.2 \%)$ in the SRS-alone group, $3(5.0 \%)$ in the Surg-SRS group, and 3

TABLE 2. Factors associated with nidus obliteration after initial SRS

\begin{tabular}{lrcccc}
\hline \multirow{2}{*}{ Variable } & \multicolumn{2}{c}{ Bivariate Analysis } & & \multicolumn{2}{c}{ Multivariate Analysis } \\
\cline { 2 - 3 } \cline { 6 - 7 } & $\mathrm{p}$ Value & $\mathrm{HR}(95 \% \mathrm{Cl})$ & & $\mathrm{p}$ Value & $\mathrm{HR}(95 \% \mathrm{Cl})$ \\
\hline Age $<30$ yrs & 0.763 & $1.04(0.82-1.30)$ & & - & - \\
\hline Male sex & 0.158 & $0.85(0.67-1.07)$ & & - & - \\
\hline Max AVM diameter $\leq 20 \mathrm{~mm}$ & $<0.001$ & $1.91(1.50-2.42)$ & & $<0.001$ & $1.81(1.43-2.30)$ \\
\hline Nidus vol $\leq 2 \mathrm{ml}$ & $<0.001$ & $1.85(1.46-2.35)$ & & - & - \\
\hline Prescription dose $\geq 20 \mathrm{~Gy}$ & $<0.001$ & $2.38(1.49-3.79)$ & & $\mathbf{0 . 0 0 3}$ & $2.04(1.28-3.27)$ \\
\hline Deep drainage & 0.170 & $0.84(0.66-1.08)$ & & 0.272 & $0.87(0.68-1.12)$ \\
\hline Eloquent location & 0.152 & $0.84(0.66-1.07)$ & & - & - \\
\hline Prior resection & $<0.001$ & $1.93(1.42-2.63)$ & & $<0.001$ & $1.78(1.30-2.43)$ \\
\hline Prior embolization & 0.291 & $0.81(0.55-1.19)$ & & 0.783 & $0.95(0.64-1.40)$ \\
\hline SMG I-II & $<0.001$ & $1.52(1.21-1.92)$ & & - & - \\
\hline mRBAS <1.0 & $<0.001$ & $0.63(0.50-0.79)$ & & - & - \\
\hline VRAS score 1-2 & $<0.001$ & $1.90(1.49-2.42)$ & & - & - \\
\hline
\end{tabular}

Boldface type indicates statistical significance $(p<0.05)$. 


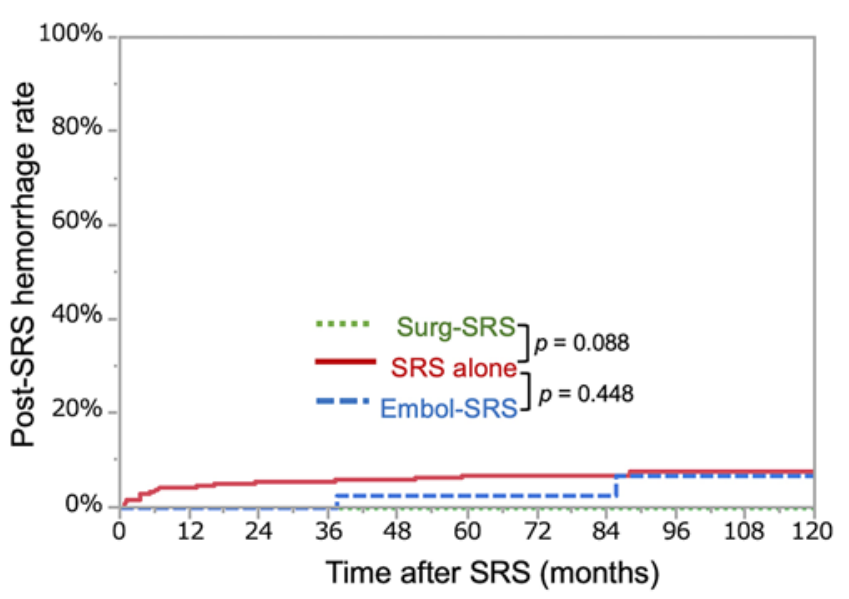

FIG. 2. Kaplan-Meier curves showing the post-SRS hemorrhage rates. Figure is available in color online only.

$(6.7 \%)$ in the Embol-SRS group. The multivariate logistic regression analysis revealed an association between a maximum AVM diameter $\leq 20 \mathrm{~mm}(\mathrm{HR} 0.42,95 \% \mathrm{CI}$ $0.19-0.95, \mathrm{p}=0.037)$ and a lower rate of symptomatic RICs (Table 4).

Delayed adverse events were observed in 20 cases (4.9\%) at a median interval of 161 months after SRS, including chronic encapsulated hematoma in 19 patients and a malignant neoplasm in 1 patient. There was no significant difference in the occurrence of delayed adverse events between the groups.

In the entire cohort, SNEs were observed in 20 cases $(4.9 \%)$, consisting of $17(5.6 \%), 1(1.7 \%)$, and $2(4.4 \%)$ patients in the SRS-alone, Surg-SRS, and Embol-SRS groups, respectively. The 10-year SNE-free rates were $95 \%$ in the entire cohort, $95 \%$ in both the SRS-alone and Embol-SRS groups, and $97 \%$ in the Surg-SRS group. No significant differences were observed in the SNE-free rates among the groups (Fig. 3). In the entire cohort, disease-specific deaths were observed in 7 patients $(1.7 \%), 6$ patients $(2.0 \%)$ in the SRS-alone group, none in the SurgSRS group, and $1(2.2 \%)$ in the Embol-SRS group. In addition, no significant difference in disease-specific deaths was observed among the groups.

\section{Matched Cohort Analysis}

Propensity score matching between the SRS-alone and Surg-SRS groups yielded matched groups with 56 patients in each group. There were no significant differences in baseline characteristics between the two groups (Supplementary Table 1). The log-rank test demonstrated that the obliteration rate was significantly higher in the Surg-SRS group than in the SRS-alone group $(\mathrm{p}=0.034)$. The postSRS hemorrhage rates $(\mathrm{p}=0.288)$ and $\mathrm{SNE}$-free rates $(\mathrm{p}=$ $0.621)$ were not significantly different.

Propensity score matching between the SRS-alone and Embol-SRS groups yielded matched groups with 40 patients in each group. There were no significant differences in baseline variables between the matched cohorts (Supplementary Table 2). There was no significant difference in the obliteration rates $(\mathrm{p}=0.423)$, post-SRS hemorrhage rates $(\mathrm{p}=0.115)$, and $\mathrm{SNE}$-free rates $(\mathrm{p}=0.430)$ between the two groups.

\section{Discussion}

This study demonstrated that SRS alone provides a favorable obliteration rate (79\% at 5 years) with a reasonable safety profile. The post-SRS hemorrhage risk in the SRS-alone group was low (1.5\% within 5 years and $0.2 \%$ thereafter), especially given that the reported annual hemorrhagic risk of untreated rAVMs ranges between $4.5 \%$ and 7.5\% ${ }^{7,22,23}$ Moreover, the decent SNE-free rate $(95 \%$ at 10 years) further substantiates the safety of SRS alone. Although numerous studies have reported AVM treatment outcomes, few recent studies have focused on rAVM, ,, $24-27$ and very few of them have described the outcomes of SRS alone for rAVM (Table 5). Hence, this study would serve as an important basis for selecting an optimal intervention for rAVM.

TABLE 3. Factors associated with post-SRS hemorrhage

\begin{tabular}{|c|c|c|c|c|}
\hline \multirow[b]{2}{*}{ Variable } & \multicolumn{2}{|c|}{ Bivariate Analysis } & \multicolumn{2}{|c|}{ Multivariate Analysis } \\
\hline & p Value & $\operatorname{HR}(95 \% \mathrm{Cl})$ & $p$ Value & $\operatorname{HR}(95 \% \mathrm{Cl})$ \\
\hline Age $<30$ yrs & 0.106 & $0.52(0.24-1.15)$ & - & - \\
\hline Male sex & 0.432 & $1.37(0.62-3.03)$ & - & - \\
\hline Max diameter $\leq 20 \mathrm{~mm}$ & 0.102 & $1.92(0.88-4.17)$ & 0.195 & $0.59(0.27-1.31)$ \\
\hline Nidus vol $\leq 2 \mathrm{ml}$ & 0.166 & $0.58(0.27-1.25)$ & - & - \\
\hline Prescription dose $\geq 20$ Gy & 0.001 & $0.25(0.11-0.57)$ & 0.007 & $0.31(0.13-0.73)$ \\
\hline Deep drainage & 0.278 & $1.72(0.65-4.55)$ & - & - \\
\hline Eloquent location & 0.193 & $1.91(0.72-5.08)$ & 0.337 & $1.62(0.23-1.65)$ \\
\hline Prior resection & 0.139 & $0.22(0.03-1.63)$ & 0.230 & $0.29(0.04-2.19)$ \\
\hline Prior embolization & 0.562 & $0.65(0.15-2.76)$ & 0.320 & $0.48(0.11-2.06)$ \\
\hline SMG I-II & 0.149 & $0.53(0.22-1.26)$ & - & - \\
\hline mRBAS $<1.0$ & 0.040 & $0.43(0.19-0.96)$ & - & - \\
\hline VRAS score 1-2 & 0.083 & $0.50(0.23-1.09)$ & - & - \\
\hline
\end{tabular}

Boldface type indicates statistical significance $(p<0.05)$. 
TABLE 4. Factors associated with symptomatic RIC

\begin{tabular}{|c|c|c|c|c|}
\hline \multirow[b]{2}{*}{ Variable } & \multicolumn{2}{|c|}{ Bivariate Analysis } & \multicolumn{2}{|c|}{ Multivariate Analysis } \\
\hline & p Value & $\mathrm{HR}(95 \% \mathrm{Cl})$ & $p$ Value & $\operatorname{HR}(95 \% \mathrm{Cl})$ \\
\hline Age $<30$ yrs & 0.847 & $1.09(0.50-2.35)$ & - & - \\
\hline Male sex & 1.000 & $0.99(0.46-2.13)$ & - & - \\
\hline Max AVM diameter $\leq 20 \mathrm{~mm}$ & 0.017 & $0.38(0.17-0.84)$ & 0.037 & $0.42(0.19-0.95)$ \\
\hline Nidus vol $\leq 2 \mathrm{ml}$ & 0.110 & $0.50(0.23-1.08)$ & - & - \\
\hline Prescription dose $\geq 20$ Gy & 0.525 & $0.70(0.23-2.12)$ & - & - \\
\hline Deep drainage & 0.086 & $2.57(0.87-7.59)$ & 0.107 & $2.45(0.82-7.31)$ \\
\hline Eloquent location & 0.217 & $1.84(0.73-4.65)$ & - & - \\
\hline Prior resection & 0.782 & $0.69(0.20-2.35)$ & 0.770 & $0.83(0.23-2.92)$ \\
\hline Prior embolization & 1.000 & $1.03(0.30-3.54)$ & 0.703 & $1.28(0.36-4.58)$ \\
\hline SMG I-II & 0.074 & $0.44(0.18-1.07)$ & - & - \\
\hline mRBAS $<1.0$ & 0.169 & $0.54(0.25-1.19)$ & - & - \\
\hline VRAS score 1-2 & 0.027 & $0.42(0.19-0.91)$ & - & - \\
\hline
\end{tabular}

Boldface type indicates statistical significance $(p<0.05)$.

It should be clarified that the emergency management of acutely ill rAVM patients has to be considered separately because patients in the current study do not directly reflect such patients. According to expert opinion, hematoma evacuation and decompression are warranted to save a life in cases of acute rupture depending on its severity. ${ }^{28} \mathrm{It}$ is probably better both to resect small, superficial, noneloquent AVMs and to embolize in an acute or intermediate fashion any obviously accessible flow-related or intranidal aneurysms. ${ }^{29,30}$ After the acute phase, definitive treatment for AVM should be performed, a situation similar to that in the current study. ${ }^{26,30}$

The main advantage of surgical treatment is the immediate reduction of hemorrhage risk. Nevertheless, a postoperative remnant is rare but possible, and a zero risk of postoperative hemorrhage is not always obtained. Surgery-related complications can matter as well. Indeed, recent surgical series have reported an $87 \%-100 \%$ chance of complete obliteration, up to a $14 \%$ chance of postsur-

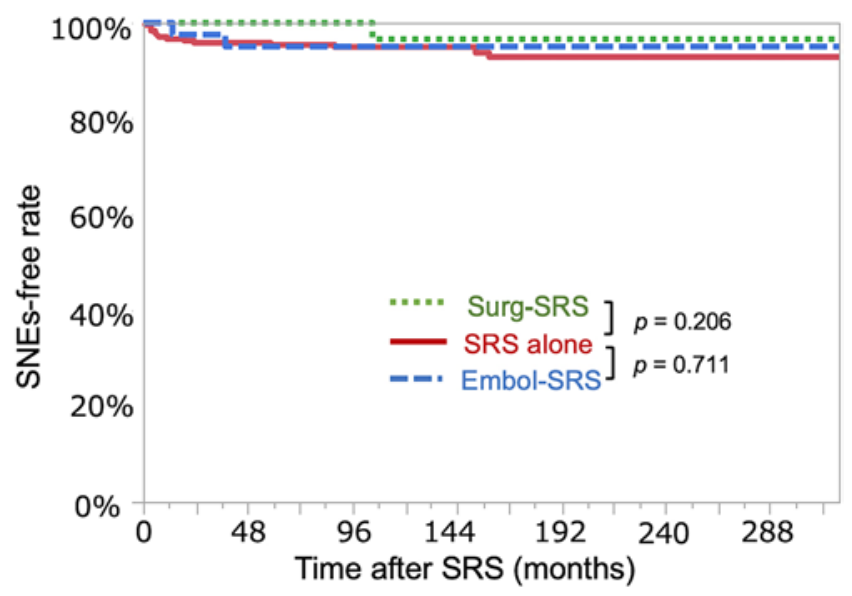

FIG. 3. Kaplan-Meier curves displaying the SNE-free rates. Figure is available in color online only. gical hemorrhage, and a 7\%-15\% chance of neurological decline. ${ }^{31-33}$ Surgical morbidities were observed in $10 \%$ of our cohort. Although SRS has been traditionally considered the second-best method for treating rAVM and has been spared for deep-seated or eloquent niduses, it seems acceptable to consider SRS as a reasonable alternative stand-alone modality given the favorable outcomes obtained in our study. In addition, the restorative use of SRS following surgery is equally feasible, as the multivariate analysis demonstrated an association between prior surgical intervention and a better chance of obliteration compared to that with SRS alone. Although there were some differences in baseline characteristics between the treatment groups, similar results were confirmed with the matched cohort analyses. The reason for the better obliteration rate in the Surg-SRS group is unclear; however, the partial disconnection of blood inflow might have impeded the intranidal flow, possibly facilitating radiation-induced intraluminal thrombosis and subsequent obliteration. On the contrary, the more that procedures are performed, the more likely treatment-related invasiveness and complications will become tangible; thus, a simpler treatment is generally better, if feasible. SRS would be applicable as a stand-alone or salvage treatment for a remnant nidus following a planned complete resection.

The interval between hemorrhage and SRS was relatively longer (10.6 months) in this study than expected. This is mainly because most of the patients were referred to us after having undergone acute care and the subsequent rehabilitation. Additionally, residual AVMs were discovered on follow-up studies performed several months after the detection of hemorrhage. Nevertheless, such findings were unlikely to impact the outcomes of SRS.

While the post-SRS hemorrhage rate in the latency phase was slightly better in the Embol-SRS group, no advantageous or disadvantageous effect was observed in multivariate analyses. In principle, embolization is an equally good alternative; the success rate has been reported to be higher than $90 \%$, especially for SMG I-II AVMs. ${ }^{34,35}$ Regarding 


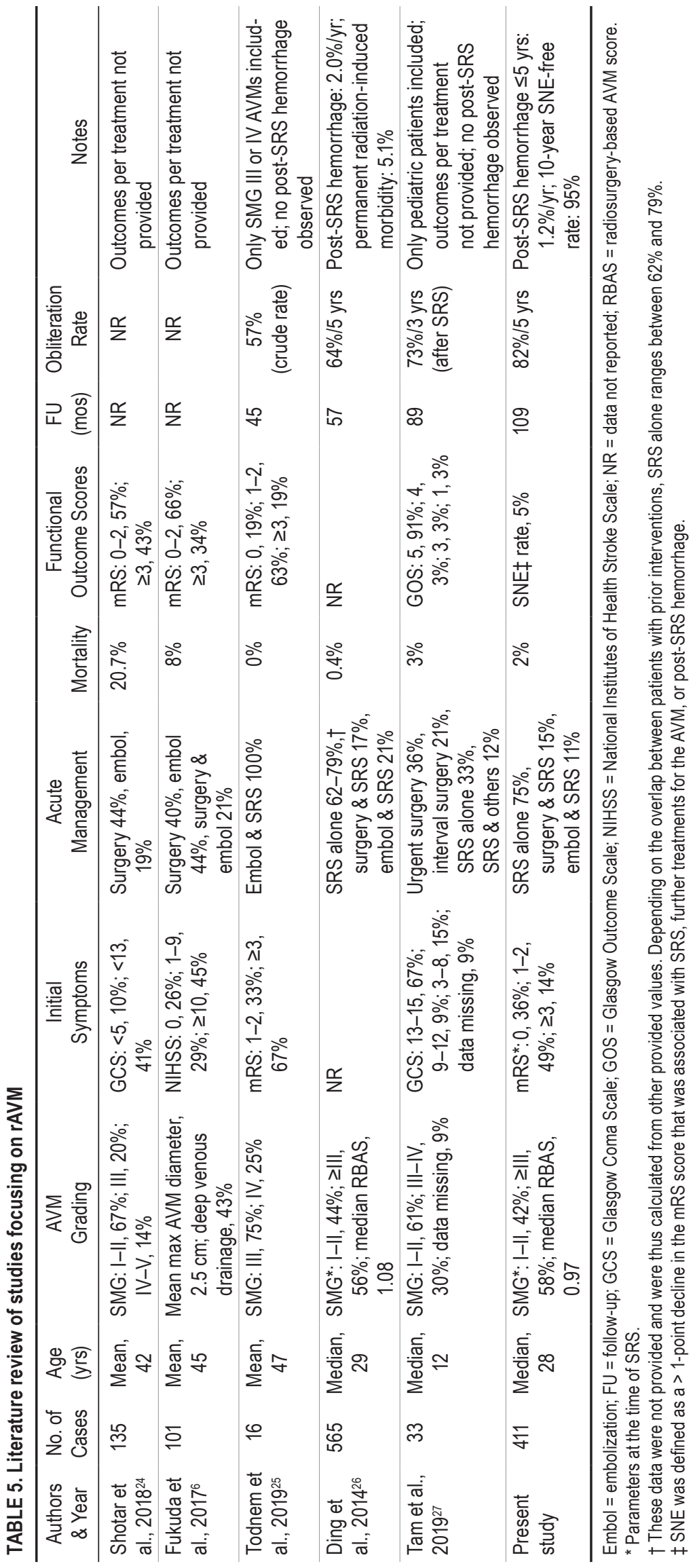


the downsides, hemorrhagic complications are possible in up to $11 \%$ of cases, postprocedural ischemic lesions can occur in up to $22 \%$ of cases, and overall morbidity and mortality can be up to $14 \%$ and $3 \%$, respectively. ${ }^{35-37}$ In the current study, several patients actually experienced morbidities as well. Embolization with SRS, initially deemed a great combination (because embolization could reduce the nidus size down to the level at which SRS could be suitably applied), eventually became questionable because the obliteration rate might be unfavorably decreased. ${ }^{38-40}$ However, this remains a matter of ongoing discussion, and this combination should not be abandoned yet. ${ }^{41}$ Indeed, in our study, obliteration rates in the Embol-SRS group were decent, although the nidus size was larger than that in the SRS-alone group. Concurrently, it is worth shifting attention (regarding embolization) from size reduction to the targeted embolization of possible bleeding points. ${ }^{42,43}$ The maximum size limit in SRS for AVM has gradually increased in the last 2 decades. It has been indicated that single-session SRS with a regular dose is safe even for a large AVM $(10-20 \mathrm{ml}){ }^{44}$ For larger AVMs (>20 ml), volume-staged SRS was adopted after the mid-2000s. ${ }^{45}$ Therefore, pre-SRS embolization aiming for volume reduction may not be as important as before; the goal of pre-SRS embolization has been shifted to targeted embolization for possible bleeding points such as an intranidal aneurysm. ${ }^{29}$

This study has several limitations. First, as a retrospective study, it is inherently subject to bias resulting from the selection of treatment modalities. To minimize the potential bias, we performed not only multivariate analysis but also matched cohort analyses, which produced similar results. Hence, the obtained results are valid and reliable. Although a prospective study is desirable to evaluate the efficacy of SRS for hemorrhagic AVMs, it would be difficult to conduct such a study given the scarcity of AVMs and the need for emergency care in such cases. Therefore, a retrospective case series including the maximum possible number of patients is the best way forward. Second, since high-grade AVMs requiring staged SRS were excluded, this study did not provide any recommendations regarding their management. Such cases would likely require a multidisciplinary treatment strategy involving the tolerance of some mild adverse effects. Third, recent evolutions in endovascular techniques were not considered because the majority of patients in the Embol-SRS group were treated before the 2000s. Similarly, the efficacy of targeted embolization could not be referenced, as there were only a few such patients in the cohort. Further case accumulation and reanalysis would therefore be desirable. Despite these limitations, this study is of scientific interest because it demonstrated the long-term outcome of SRS for rAVM per treatment modality-not only as a sole treatment modality, but also as a salvage treatment after failed resection or embolization.

\section{Conclusions}

Although the definitive treatment for RAVM should be determined after due consideration of a patient's characteristics, AVM radiographic features, and in-facility circumstances, SRS alone would be a reasonable option, as evidenced by the provision of a 79\% 5-year obliteration rate, $1.5 \%$ annual latency phase hemorrhage rate, and $95 \%$ 10-year SNE-free rate. SRS can also be favorably used for residual AVMs after initial interventions; notably, excellent results were achieved in AVMs for which resection had failed.

\section{References}

1. Friedlander RM. Clinical practice. Arteriovenous malformations of the brain. N Engl J Med. 2007;356(26):2704-2712.

2. Choi JH, Mohr JP. Brain arteriovenous malformations in adults. Lancet Neurol. 2005;4(5):299-308.

3. Brown RD Jr, Wiebers DO, Forbes G, et al. The natural history of unruptured intracranial arteriovenous malformations. J Neurosurg. 1988;68(3):352-357.

4. ApSimon HT, Reef H, Phadke RV, Popovic EA. A population-based study of brain arteriovenous malformation: longterm treatment outcomes. Stroke. 2002;33(12):2794-2800.

5. Choi JH, Mast H, Sciacca RR, et al. Clinical outcome after first and recurrent hemorrhage in patients with untreated brain arteriovenous malformation. Stroke. 2006;37(5):12431247.

6. Fukuda K, Majumdar M, Masoud H, et al. Multicenter assessment of morbidity associated with cerebral arteriovenous malformation hemorrhages. J Neurointerv Surg. 2017;9(7): 664-668.

7. Pollock BE, Flickinger JC, Lunsford LD, et al. Factors that predict the bleeding risk of cerebral arteriovenous malformations. Stroke. 1996;27(1):1-6.

8. Stapf C, Mast H, Sciacca RR, et al. Predictors of hemorrhage in patients with untreated brain arteriovenous malformation. Neurology. 2006;66(9):1350-1355.

9. Yen CP, Sheehan JP, Schwyzer L, Schlesinger D. Hemorrhage risk of cerebral arteriovenous malformations before and during the latency period after GAMMA knife radiosurgery. Stroke. 2011;42(6):1691-1696.

10. Mohr JP, Parides MK, Stapf C, et al. Medical management with or without interventional therapy for unruptured brain arteriovenous malformations (ARUBA): a multicentre, nonblinded, randomised trial. Lancet. 2014;383(9917):614-621.

11. Al-Shahi Salman R, White PM, Counsell CE, et al. Outcome after conservative management or intervention for unruptured brain arteriovenous malformations. JAMA. 2014; 311(16):1661-1669.

12. Schneider BF, Eberhard DA, Steiner LE. Histopathology of arteriovenous malformations after gamma knife radiosurgery. J Neurosurg. 1997;87(3):352-357.

13. Karlsson B, Lax I, Söderman M. Risk for hemorrhage during the 2-year latency period following gamma knife radiosurgery for arteriovenous malformations. Int J Radiat Oncol Biol Phys. 2001;49(4):1045-1051.

14. Maruyama K, Kawahara N, Shin M, et al. The risk of hemorrhage after radiosurgery for cerebral arteriovenous malformations. N Engl J Med. 2005;352(2):146-153.

15. Hanakita S, Shin M, Koga T, et al. Risk reduction of cerebral stroke after stereotactic radiosurgery for small unruptured brain arteriovenous malformations. Stroke. 2016;47(5):12471252.

16. Shin M, Maruyama K, Kurita H, et al. Analysis of nidus obliteration rates after gamma knife surgery for arteriovenous malformations based on long-term follow-up data: the University of Tokyo experience. J Neurosurg. 2004;101(1):18-24.

17. Hasegawa H, Hanakita S, Shin M, et al. A comprehensive study of symptomatic late radiation-induced complications after radiosurgery for brain arteriovenous malformation: incidence, risk factors, and clinical outcomes. World Neurosurg. 2018;116:e556-e565. 
18. Pollock BE, Flickinger JC. Modification of the radiosurgerybased arteriovenous malformation grading system. Neurosurgery. 2008;63(2):239-243.

19. Spetzler RF, Martin NA. A proposed grading system for arteriovenous malformations. J Neurosurg. 1986;65(4):476-483.

20. Starke RM, Yen CP, Ding D, Sheehan JP. A practical grading scale for predicting outcome after radiosurgery for arteriovenous malformations: analysis of 1012 treated patients. $\mathrm{J} \mathrm{Neu-}$ rosurg. 2013;119(4):981-987.

21. Kawashima M, Hasegawa H, Kurita H, et al. Ectopic recurrence of arteriovenous malformation after radiosurgery: case report and insight regarding pathogenesis. World Neurosurg. 2020;135:63-67.

22. Gross BA, Du R. Natural history of cerebral arteriovenous malformations: a meta-analysis. J Neurosurg. 2013;118(2): 437-443.

23. da Costa L, Wallace MC, Ter Brugge KG, et al. The natural history and predictive features of hemorrhage from brain arteriovenous malformations. Stroke. 2009;40(1):100-105.

24. Shotar E, Debarre M, Sourour NA, et al. Retrospective study of long-term outcome after brain arteriovenous malformation rupture: the RAP score. J Neurosurg. 2018;128(1):78-85.

25. Todnem N, Ward A, Nahhas M, et al. A retrospective cohort analysis of hemorrhagic arteriovenous malformations treated with combined endovascular embolization and Gamma Knife stereotactic radiosurgery. World Neurosurg. 2019;122:e713e722.

26. Ding D, Yen CP, Starke RM, et al. Radiosurgery for ruptured intracranial arteriovenous malformations. J Neurosurg. 2014; 121(2):470-481.

27. Tam KY, Lim K, Zhu CXL, et al. Long-term outcomes of ruptured cerebral arteriovenous malformations in the paediatric population: a retrospective review in a regional hospital in Hong Kong. J Clin Neurosci. 2019;66:66-70.

28. Kato Y, Dong VH, Chaddad F, et al. Expert consensus on the management of brain arteriovenous malformations. Asian J Neurosurg. 2019;14(4):1074-1081.

29. Kano H, Kondziolka D, Flickinger JC, et al. Aneurysms increase the risk of rebleeding after stereotactic radiosurgery for hemorrhagic arteriovenous malformations. Stroke. 2012; 43(10):2586-2591.

30. Ding D, Chen CJ, Starke RM, et al. Risk of brain arteriovenous malformation hemorrhage before and after stereotactic radiosurgery. Stroke. 2019;50(6):1384-1391.

31. Korja M, Bervini D, Assaad N, Morgan MK. Role of surgery in the management of brain arteriovenous malformations: prospective cohort study. Stroke. 2014;45(12):3549-3555.

32. Schramm J, Schaller K, Esche J, Boström A. Microsurgery for cerebral arteriovenous malformations: subgroup outcomes in a consecutive series of 288 cases. J Neurosurg. 2017; 126(4):1056-1063.

33. Theofanis T, Chalouhi N, Dalyai R, et al. Microsurgery for cerebral arteriovenous malformations: postoperative outcomes and predictors of complications in 264 cases. Neurosurg Focus. 2014;37(3):E10.

34. van Rooij WJ, Jacobs S, Sluzewski M, et al. Curative embolization of brain arteriovenous malformations with onyx: patient selection, embolization technique, and results. AJNR Am J Neuroradiol. 2012;33(7):1299-1304.

35. Katsaridis V, Papagiannaki C, Aimar E. Curative embolization of cerebral arteriovenous malformations (AVMs) with Onyx in 101 patients. Neuroradiology. 2008;50(7):589-597.

36. Baharvahdat H, Blanc R, Termechi R, et al. Hemorrhagic complications after endovascular treatment of cerebral arteriovenous malformations. AJNR Am J Neuroradiol. 2014; 35(5):978-983.
37. Saatci I, Geyik S, Yavuz K, Cekirge HS. Endovascular treatment of brain arteriovenous malformations with prolonged intranidal Onyx injection technique: long-term results in 350 consecutive patients with completed endovascular treatment course. J Neurosurg. 2011;115(1):78-88.

38. Andrade-Souza YM, Ramani M, Scora D, et al. Embolization before radiosurgery reduces the obliteration rate of arteriovenous malformations. Neurosurgery. 2007;60(3):443-452.

39. Kano H, Kondziolka D, Flickinger JC, et al. Stereotactic radiosurgery for arteriovenous malformations after embolization: a case-control study. J Neurosurg. 2012;117(2):265-275.

40. Pollock BE, Flickinger JC, Lunsford LD, et al. Factors associated with successful arteriovenous malformation radiosurgery. Neurosurgery. 1998;42(6):1239-1247.

41. Hasegawa H, Yamamoto M, Shin M, Barfod BE. Gamma knife radiosurgery for brain vascular malformations: current evidence and future tasks. Ther Clin Risk Manag. 2019;15: 1351-1367.

42. Alexander MD, Hippe DS, Cooke DL, et al. Targeted embolization of aneurysms associated with brain arteriovenous malformations at high risk for surgical resection: a case-control study. Neurosurgery. 2018;82(3):343-349.

43. Sun Y, Jin H, Li Y, Tian Z. Target embolization of associated aneurysms in ruptured arteriovenous malformations. World Neurosurg. 2017;101:26-32.

44. Hasegawa H, Hanakita S, Shin M, et al. Re-evaluation of the size limitation in single-session stereotactic radiosurgery for brain arteriovenous malformations: detailed analyses on the outcomes with focusing on radiosurgical doses. Neurosurgery. 2020;86(5):685-696.

45. Hanakita S, Shin M, Koga T, et al. Outcomes of volumestaged radiosurgery for cerebral arteriovenous malformations larger than $20 \mathrm{~cm}^{3}$ with more than 3 years of follow-up. World Neurosurg. 2016;87:242-249.

\section{Disclosures}

The authors report no conflicts of interest concerning the materials or methods used in this study or the findings specified in this paper.

\section{Author Contributions}

Conception and design: Kawashima, Hasegawa. Acquisition of data: Kawashima, Ishikawa, Koizumi, Katano. Analysis and interpretation of data: Kawashima, Hasegawa. Drafting the article: Kawashima. Critically revising the article: Hasegawa, Shin, Shinya. Reviewed submitted version of manuscript: Shin, Shinya, Ishikawa, Koizumi, Katano. Study supervision: Shin, Nakatomi, Saito.

\section{Supplemental Information Online-Only Content}

Supplemental material is available with the online version of the article.

Supplementary Tables 1 and 2. https://thejns.org/doi/suppl/ 10.3171/2020.7.JNS201502.

\section{Correspondence}

Mariko Kawashima: University of Tokyo Hospital, Tokyo, Japan. mrkawashima-tky@umin.ac.jp. 